\title{
Enhancing the ratio of fluorescence to bremsstrahlung radiation in X-ray tube spectra
}

\author{
L.M.N. Távora ${ }^{\mathrm{a}, *}$, E.J. Morton ${ }^{\mathrm{b}}$, W.B. Gilboy ${ }^{\mathrm{b}}$ \\ Departamento de Física, Universidade de Coimbra, 3004-516 Coimbra, Portugal \\ ${ }^{\mathrm{b}}$ Physics Department, University of Surrey, Guildford, Surrey GU2 $5 X H, U K$
}

Received 18 October 1999; received in revised form 19 January 2000; accepted 25 January 2000

\begin{abstract}
This paper describes techniques that can be used to improve the ratio of fluorescence to bremsstrahlung radiation $(F / B)$ in X-ray tube spectra. Firstly, an extension of the EGS4 code system is used to evaluate the impact of the substrate in thin target applications, in terms of the yield of bremsstrahlung photons produced. The choice of materials to filter X-ray tube spectra, and its effect in the $F / B$ and the tube efficiency is discussed. The characteristics of spectra produced in transmission tubes with different target thicknesses, substrates and tube voltages are also presented. (C) 2000 Elsevier Science Ltd. All rights reserved.
\end{abstract}

Keywords: Fluoresence radiation; Bremsstrahlung; X-ray tube spectra

\section{Introduction}

A wide variety of imaging applications rely on X-ray tubes as the source of ionising radiation. As an alternative, radioisotope sources can be employed but usually their brightness is several orders of magnitude less than that obtained with X-ray tubes. Additionally, radioactive sources may not be turned off. However, $\mathrm{X}$-ray tubes emit photons in a broad range of energies rather than at one or more discrete lines, and this may be regarded as a major limitation for the purpose of accurately determining subtle changes in photon attenuation coefficient. Therefore, detailed studies that aimed to understand the fundamental processes re-

* Corresponding author. Tel.: +351-239410667; fax: +351239829158.

E-mail address: lmtavora@saturno.fis.uc.pt (L.M.N. Távora). sponsible for the conversion of the electron's kinetic energy into radiated photons in X-ray tubes are of some importance. The operation of X-ray tubes can then be understood at a detailed level and the device optimised for a particular imaging application.

The importance of accurately predicting X-ray tube spectra has been recognized since the early work of Kramers (1920) and from then several approaches have been described in the literature. These include semiempirical (Sundararaman et al., 1973; Birch and Marshall, 1979; Tucker et al., 1991) and numerical (Boone and Seibert, 1997) methods as well as reconstructive algorithms (Archer and Wagner, 1998) that can reproduce X-ray tube spectra realistically. References to further work on this topic can be found in publications of Boone and Seibert (1997) and Távora et al. (1999a), while benchmarks of different models against experimental data have been provided by Bissonete and Schreiner (1992) and Bhat et al. (1998a, 1998b). 
It is nowadays acknowledged that the Monte Carlo method is a powerful technique for simulating the transport of radiation in matter (Jenkins et al., 1988; Andreo, 1991). Moreover, the increase in computer power along with a dramatic fall in cost (Távora and Morton, 1998), has prompted the development of different general purpose codes (Seltzer, 1991; Nelson et al., 1985; Briesmeister, 1997). One of the packages that can be used to simulate the coupled transport of electrons, positrons and photons in matter is the EGS4 code system (Nelson et al., 1985). This powerful and versatile piece of software was originally developed for high energy physics applications, but several improvements have been implemented, and the code can now be used down to energies of the order of $10 \mathrm{keV}$ for charged particles and $1 \mathrm{keV}$ for photons (Nelson et al., 1997).

Recently (Távora and Morton, 1998), we described a low energy electron extension of EGS4, especially suited to simulate the production of radiation in X-ray tubes operating below $100 \mathrm{kV}_{\mathrm{p}}$. This enhanced code (briefly called EGS4/GOS) includes more realistic models for the production of fluorescence radiation and for generating the angular distribution of bremsstrahlung photons. The code was used to simulate transmission target $\mathrm{X}$-ray tubes and benchmarked against experimental data. The simulated spectra were found to be in good agreement with experimental results, with an accuracy of the order of $10 \%$ at the $K_{\alpha}$ line, for different tube voltages and target thicknesses.

In a further communication (Távora et al., 1999a), the EGS4/GOS code was used to gain some insight into the physics of $\mathrm{X}$-ray tubes mounted in transmission geometry, when operated at diagnostic energies. The depth at which the radiation is produced, as well as its angular distribution, was evaluated and we concluded that the spectral properties of transmitted spectra depend on the thickness of the target. In the case of Ag targets, we showed that spectra produced in thinner targets exhibit higher fluorescence to bremsstrahlung ratios. However this improvement is paid for at the expense of a fall in the total number of fluorescence photons produced in a given exposure. In other words, the target thickness in transmission X-ray tubes can be chosen so that 'quasi-monoenergetic' spectra are produced although this is at the expense of the tube's efficiency.

More recently (Távora et al., 1999a), we also demonstrated that for energies near the target $K$-edge higher tube voltages clearly favor the emission of fluorescence photons over bremsstrahlung radiation. This happens not only because the cross section for the ionisation of the $K$-shell by electron impact increases more sharply than that for bremsstrahlung emissions (Távora et al., 1999a), but also because there is more energy 'available', making it more likely to ionise the $K$-shell even after some of the electron's incident energy has been lost in inelastic processes (atomic excitation electron-electron collisions and radiative emissions).

In this paper, we describe additional techniques to increase the ratio of fluorescence to bremsstrahlung radiation in X-ray tube spectra. Firstly, the impact of the substrate's composition on the overall performance of thin target devices is discussed. As electrons lose only a fraction of their energy in the tube's target, it is quite conceivable that some bremsstrahlung will be produced in the substrate, thus decreasing the observed ratio of fluorescence to bremsstrahlung radiation. The choice of filter materials that act to increase the number of fluorescence photons over those due to bremsstrahlung interactions is also discussed for spectra produced in $\mathrm{Ag}$, Mo and $\mathrm{W}$ targets. Finally, the performance of different transmission X-ray tubes is compared, in terms of the dependence of their spectral properties on the tube voltage, target thickness and the film substrate.

\section{The relevance of substrate material in thin target applications}

As mentioned previously, in applications that involve the production of radiation in thin targets only a small fraction of the electron's initial energy is lost in the tube target. Therefore, it is quite reasonable to assume that some bremsstrahlung radiation will be produced in the substrate on which the target film is deposited. From the dependence of the bremsstrahlung cross section on the medium's atomic number and the incident electron energy (Jauch and Rohrlich, 1976; Perkins et al., 1991), it is understandable that this will be particularly important for thinner targets, higher electron energies and higher $Z$ substrates.

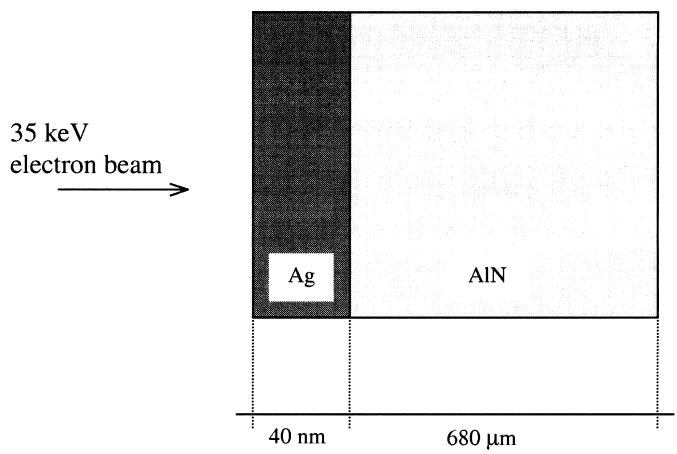

Fig. 1. Diagram showing the model used to evaluate the significance of bremsstrahlung production in the target substrate. 


\subsection{Bremsstrahlung production in AlN}

In order to gain a quantitative view on the relevance of the substrate on the whole performance of the tube, a setup consisting of a $40 \mathrm{~nm} \mathrm{Ag} \mathrm{tar-}$ get deposited onto an $680 \mu \mathrm{m}$ thick AlN substrate (Fig. 1) was considered. The bremsstrahlung production in both target and substrate, as generated by a $35 \mathrm{keV}$ electron beam incident on the target, was evaluated using the EGS4/GOS code (Távora and Morton, 1998) and the result is presented in Fig. 2. These data show that, for energy spectral measurements at $0^{\circ}$ (as detected by a sensor of 1.5 $\mathrm{cm}$ radius that is positioned $15 \mathrm{~cm}$ away from the tube), the contribution of radiative events taking place in AlN is considerably higher than those from the Ag target. It was also shown that $99 \%$ of the electrons incident on the $\mathrm{Ag}$ film are transmitted to the AlN substrate with energies above $34 \mathrm{keV}$. In this way, for the purpose of understanding bremsstrahlung production in substrates, it can be considered that a 'quasi-monoenergetic' $35 \mathrm{keV}$ electron beam is incident on the substrate.

Generally speaking, Fig. 2 suggests that the production of bremsstrahlung radiation in the target substrate may seriously degrade the performance of the tube. Indeed, although we have shown that a higher ratio of fluorescence to bremsstrahlung radiation is attainable in tubes with thinner targets (Távora et al., 1999a), a significant number of bremsstrahlung photons will still be produced in the metal's substrate. To overcome this problem (at least partially) alternative solutions are discussed in the next section.

\subsection{Alternative materials}

In broad terms, the materials chosen to be used as target substrates in transmission geometry X-ray tubes should combine good thermal conductivity with high photon transmission in the spectral region of interest (usually the target's $K_{\alpha}$ and $K_{\beta}$ lines). These characteristics provide extended tube lifetime by allowing efficient heat dissipation and so a high output flux. Moreover, in light of the results presented in Fig. 2, substrates should have low atomic numbers, thus reducing the fraction of electron energy converted into bremsstrahlung radiation. A feasible choice is to employ boron $\left(Z=5, \rho=2.34 \mathrm{~g} \mathrm{~cm}^{-3}\right)$ instead of AlN; although more challenging from the experimental point of view, lithium $\left(Z=3, \rho=0.53 \mathrm{~g} \mathrm{~cm}^{-3}\right)$ may also represent a further option, so these two materials have been considered in this study.

The bremsstrahlung yield (in the form of a ratio of the bremsstrahlung cross section to the total cross section for interactions involving energy losses) for lithium (Li), boron (B) and AlN have been calculated using the EEDL database (Perkins et al., 1991). The results are shown in Fig. 3 for electron energies of a few tens of $\mathrm{keV}$. This data indicates that substantial reductions in the number of bremsstrahlung photons generated may be obtained if $\mathrm{Li}$ or $\mathrm{B}$, rather than AlN, are used as substrates.

The geometry shown in Fig. 1 was then considered in two different configurations, with 5 and $1 \mathrm{~mm}$ thick substrates of $\mathrm{Li}$ and $\mathrm{B}$ (respectively) replacing the AlN. The amount of bremsstrahlung radiation produced in the new substrates was evaluated using the EGS4/GOS code and the results are shown in Fig. 4. This data

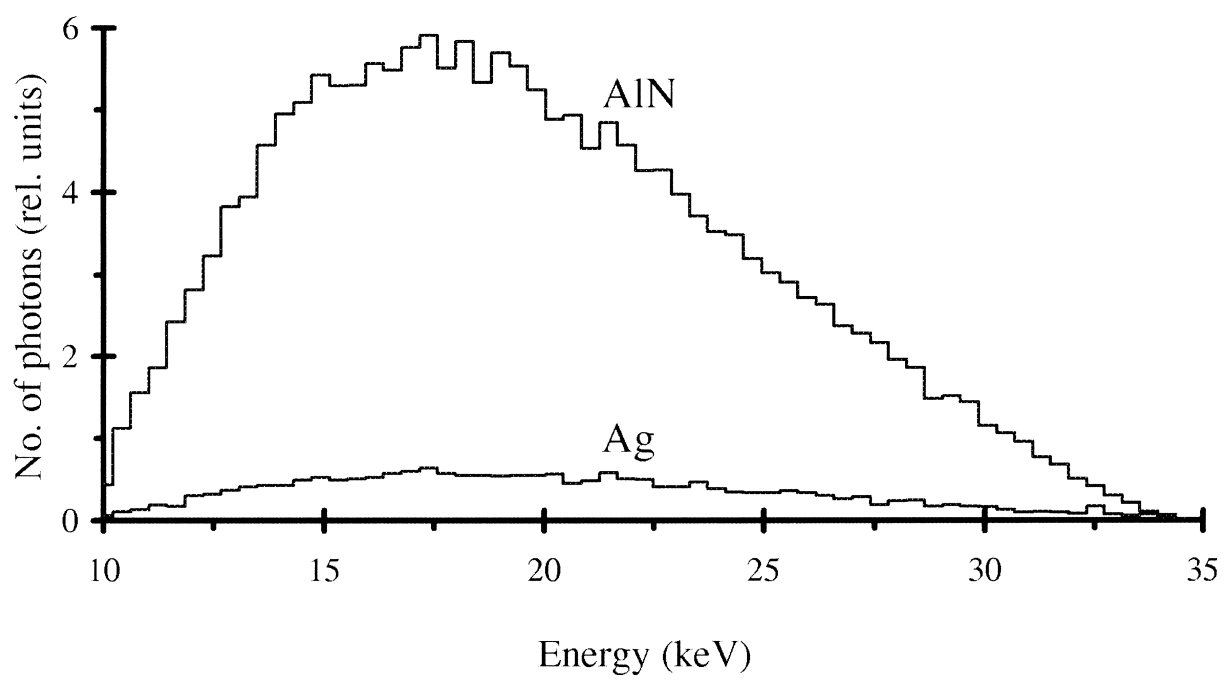

Fig. 2. Production of bremsstrahlung radiation in the Ag target and the AlN substrate. Photon spectra transmitted along the axis of the electron beam, for the geometry presented in Fig. 1. 


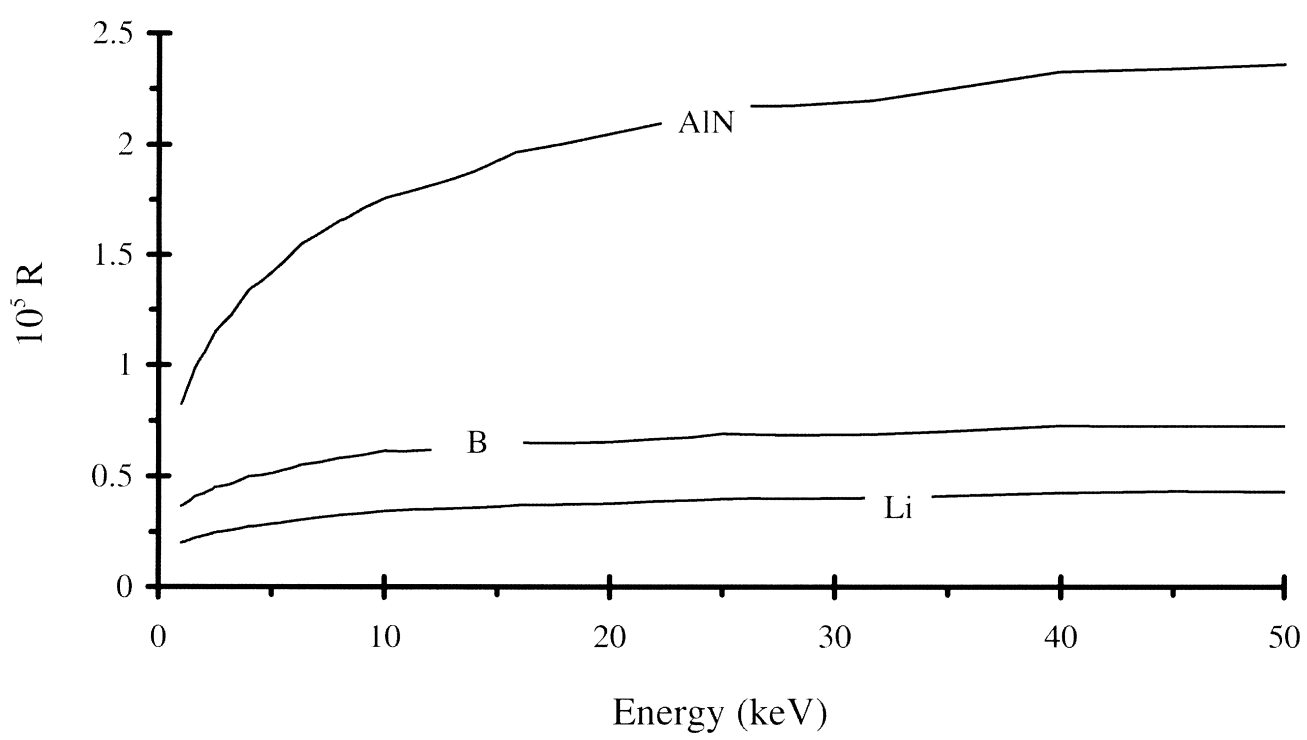

Fig. 3. Ratio $(R)$ of the bremsstrahlung cross section to the total cross section for events involving energy loss (bremsstrahlung + ionisation + excitation). Data was calculated using the EEDL database (Perkins et al., 1991).

shows that, from energies of the order of $17 \mathrm{keV}$, the contribution of bremsstrahlung corresponding to radiative emissions that take place in the substrate can be significantly reduced if $\mathrm{Li}$ or $\mathrm{B}$ replace $\mathrm{AlN}$. The enhancement of the tails at lower energies reflects the reduction in self-attenuation in $\mathrm{Li}$ and $\mathrm{B}$. In fact, low $Z$ media where bremsstrahlung interactions are less likely to occur, also turn out to be more transparent especially at lower photon energies. This is shown in Fig. 5, where photon transmission through the three substrates considered is plotted.
In summary, applications that require the use of thin Ag targets can benefit if the metal film is deposited onto a $\mathrm{B}$ or $\mathrm{Li}$ substrate, rather than on AlN. This is because a considerable reduction in the bremsstrahlung yield can be achieved. However, AlN seems rather appropriate for the purpose of filtering spectra from $\mathrm{Ag}$ targets, as it combines high photon transmission at $22.1 \mathrm{keV}(\approx 70 \%)$ with a strong absorption below $10 \mathrm{keV}(>95 \%)$. Therefore, in practical situations where $\mathrm{B}$ or $\mathrm{Li}$ are to be employed as substrates, additional filters that reduce the number of low energy

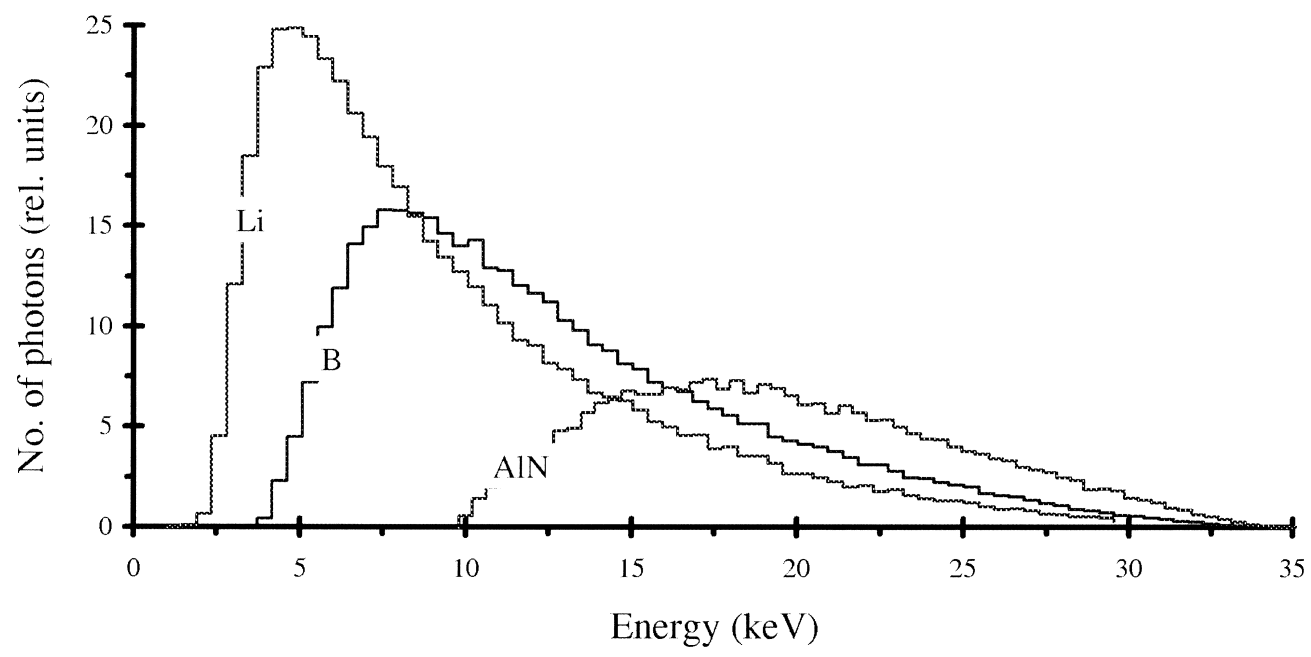

Fig. 4. Simulated photon spectra corresponding to the bremsstrahlung radiation produced in three different substrates. The geometry considered is the one shown Fig. 1, but with $\mathrm{Li}$ and B replacing AlN. Data sets are normalised to the same number of incident particles. 
photons being emitted from the tube should be considered. The choice of adequate filtering systems is discussed in the next section. In thick target applications the use of low $Z$ substrates should bring no operational advantages as very little bremsstrahlung is produced in the substrate. On the other hand, as previously analyzed, the higher photon transparencies of low $Z$ materials make them rather unsuitable for the purpose of increasing the observed ratios of fluorescence to bremsstrahlung radiation.

\section{Choosing the appropriate filter material}

The use of one or more elements to filter polychromatic spectra is a technique widely used in the field of radiation imaging (Beaman and Lillicrap, 1982; Sandborg et al., 1994a; Massoumzadeh et al., 1998). In broad terms, the purpose of this procedure is to optimise the spectral purity of an inspecting radiation. This technique is similar to those used in region of interest imaging (Rudin and Bednarek, 1993; Massoumzadeh et al., 1998) where the number of incident photons in a particular energy window is enhanced while that outside that region is decreased. Other aspects, such as the reduction of low energy photons that may (in the field of medical imaging) have undesirable side effects (like skin burn) and contribute very little to the image formation (Sandborg et al., 1994b; Martin et al., 1999) will not be addressed in the methodology presented herein.

\subsection{Method}

The optimisation of X-ray tube spectra towards the development of 'quasi-monochromatic' beams may be described (in this context and to first order) as the process that aims to enhance the ratio of fluorescence to bremsstrahlung radiation. Starting with a digitised spectrum divided into $M$ energy intervals (bins), and characterised by a distribution of $n_{i}\left(E_{i}\right)$ photons in the energy bin centered at each energy $E_{i}$, the quantity to be optimised, $F / B$, may be defined as

$F / B=\frac{n_{K}\left(E_{K}\right)}{\sum_{i \neq k} n_{i}\left(E_{i}\right)}$,

where $n_{K}\left(E_{K}\right)$ denotes the number of photons in the energy interval that corresponds to the $K_{\alpha}$ line. The quantity $F / B$ does not strictly reproduce the ratio of fluorescence to bremsstrahlung radiation in the spectrum as some of the counts in $n_{K}\left(E_{K}\right)$ actually correspond to bremsstrahlung photons. Nevertheless, this ratio can be used as a reliable figure to describe the purity of the spectrum provided that the energy bins are kept small and the $K_{\alpha}$ line is considered to be the signal to be used in the measurement. Additionally, $F /$ $B$ as defined in Eq. (1) is relatively easy to calculate, while the calculation of the 'true' ratio of fluorescence to bremsstrahlung radiation would require the estimation of the number of bremsstrahlung photons in the bin corresponding to the $K_{\alpha}$ peak, so that this quantity could then be subtracted from the observed peak.

The introduction of an element that will act to filter the original beam, results in changes in the spectral

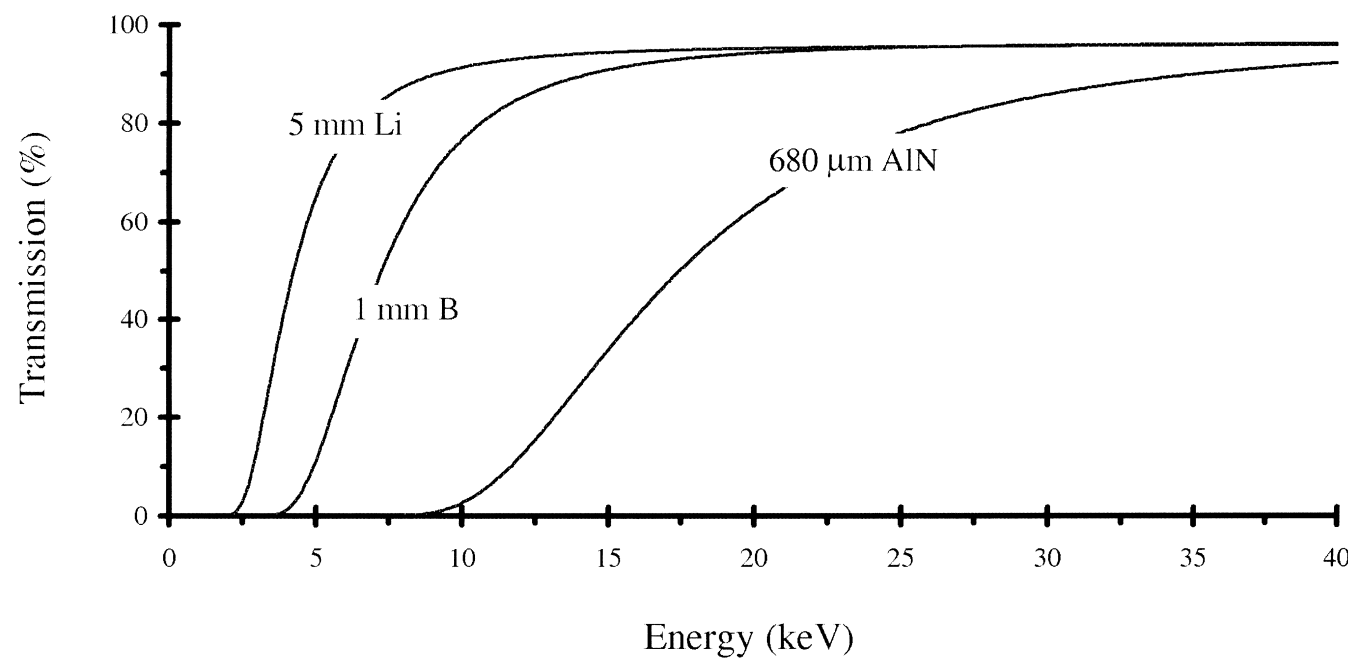

Fig. 5. Photon transmission through the three substrates considered. Data has been calculated using the XCOM program (Berger and Hubbell, 1987). 
composition of the beam, and consequently on $F / B$. Following the definition introduced above, the new ratio, $(F / B)^{\prime}$ can be calculated from

$(F / B)^{\prime}=\frac{n_{K}^{\prime}\left(E_{K}\right)}{\sum_{i \neq k} n_{i}^{\prime}\left(E_{i}\right)}$,

where $n_{i}^{\prime}\left(E_{i}\right)$ is the number of photon counts (per bin) in the transmitted beam. Assuming that all the photons have the same attenuating path $d$ in the filter (i.e., the angular spread of the beam is not significant) then

$n_{i}^{\prime}\left(E_{i}\right)=n_{i}\left(E_{i}\right) \mathrm{e}^{-\mu\left(E_{i}\right) d}$

with $\mu\left(E_{i}\right)$ denoting the linear absorption coefficient of the selected filter material, at each energy $E_{i}$. The filter should then be chosen so that $(F / B)^{\prime}$ is actually higher than $F / B$ in the original spectrum. This method will always result in a certain attenuation of the 'signal' $n_{K}\left(E_{K}\right)$, so the gains in $F / B$ should always be balanced against the intrinsic loss in efficiency. The resulting improvement in $F / B$ is expected to exhibit a strong dependence on the spectral distribution used, especially in terms of the energy of the $K_{\alpha}$ line (i.e., the type of target where the radiation is produced).

To start with, consider the spectrum produced by an $\mathrm{X}$-ray tube with an $\mathrm{Ag}$ target. The transmission geometry used is the one presented in Fig. 1, with a 40 $\mathrm{nm} \mathrm{Ag} \mathrm{film} \mathrm{deposited} \mathrm{onto} \mathrm{a} 680 \mu \mathrm{m}$ thick AlN substrate. The spectrum considered is the one presented in a previous publication (Távora and Morton, 1998), and it basically corresponds to the sum of the two data sets presented in Fig. 2 with the fluorescence radiation also considered. The program MUCAL (Boyanov, 1996), a fit to the data published by McMaster et al. (1969), was used to estimate photon attenuation coefficients and, from Eq. (3), the spectral distribution of beams transmitted through different absorbers were calculated. Then, using Eq. (2), the ratios $(F / B)^{\prime}$ were evaluated and the results are presented in Fig. 6 . The data shows the values of $F / B$ obtained with different filter materials, for two attenuations at $22.1 \mathrm{keV}\left(K_{\alpha}\right.$ line in $\mathrm{Ag}$ ), and with $F / B$ normalised to the value of the initial spectrum (with no filtration). In this way, the data points with $F / B$ above one can be understood as filters that produce a 'cleaner' spectrum.

From Fig. 6, several important conclusions can be inferred. Firstly, it is clear that some materials are better than others in terms of the improvements in $F / B$. In some cases, there may actually be a decrease in $F / B$ indicating that there was a loss in terms of spectral purity and so the material should not be used for this purpose. It can also be noticed that the filters with higher enhancements in terms of $F / B$ are those based on materials whose $K$-edge is just above the $K_{\alpha}$ line of the spectrum used (where the photon attenuation cross section reaches a minimum). On the other hand, materials with a $K$-edge just below the spectrum's $K_{\alpha}$ line are to be avoided as they strongly absorb the $K_{\alpha}$ photon signal. In the case of $\mathrm{Ag}$ spectra $\left(E_{K_{\alpha}}=22.16\right.$ $\mathrm{keV}$ ), rhodium ( $\mathrm{Rh}, Z=45, K$-edge: $23.22 \mathrm{keV}$ ) and palladium (Pd, $Z=46, K$-edge: $24.35 \mathrm{keV})$ are the best choices, with Ag itself representing a feasible alternative. It is also seen that the improvements obtained in $F / B$ are a function of the attenuation at the $K_{\alpha}$ energy

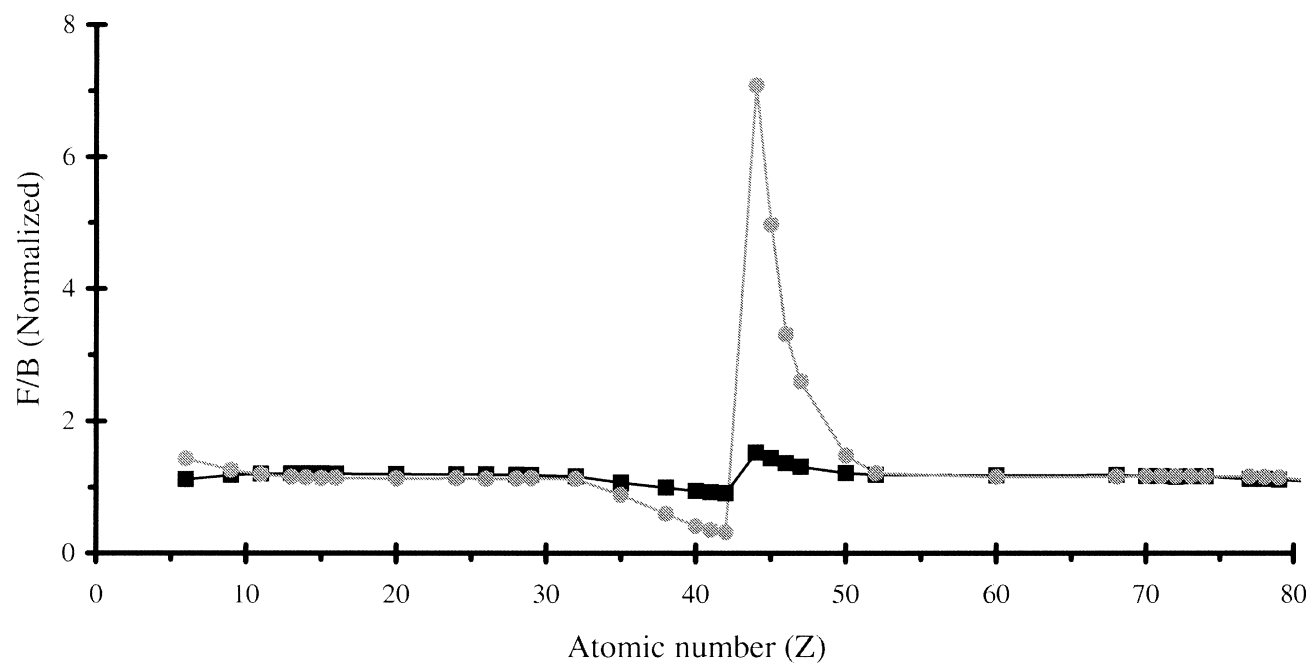

Fig. 6. Dependence of $F / B$ on the atomic number of the filter material selected, for an $\mathrm{Ag}$ spectrum. The values of $F / B$ plotted are normalized to that in the original spectrum and the two data sets correspond to attenuations of $20 \%$ (squares) and $90 \%$ (circles) at $22.1 \mathrm{keV}$. 


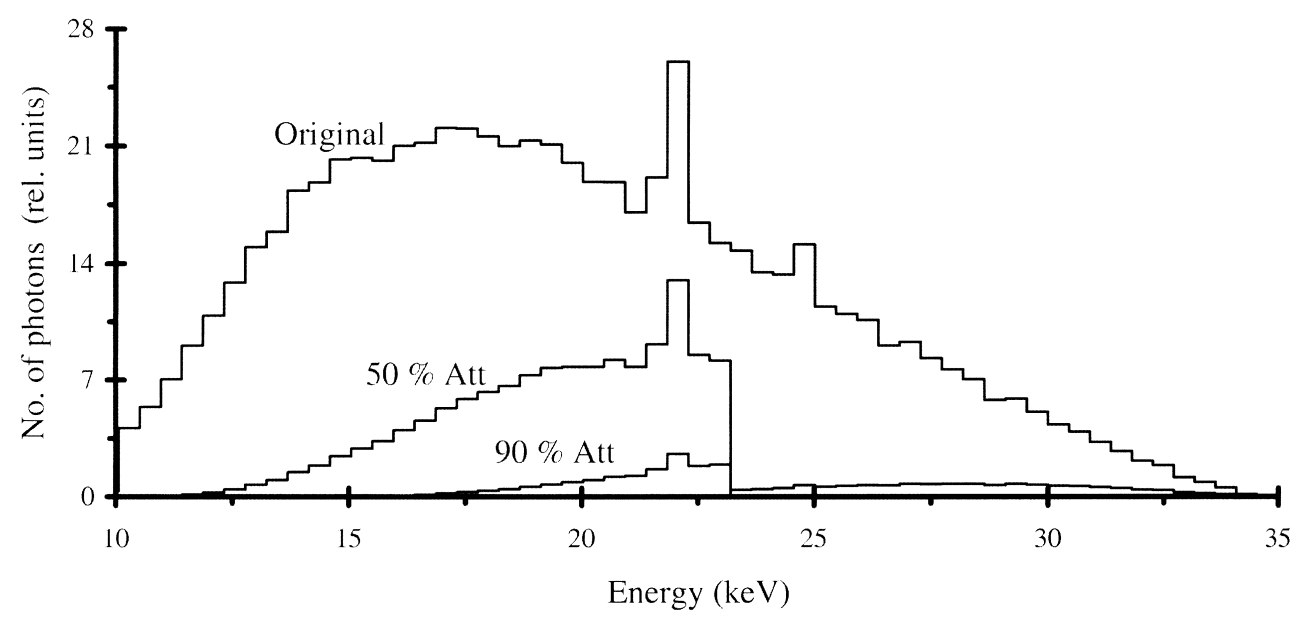

Fig. 7. Photon spectra produced in the Ag target. The data sets shown correspond to the original spectrum and those transmitted through Rh filters (with attenuations of 50 and $90 \%$ at $22.1 \mathrm{keV}$ ).

where, higher attenuations seem to result in more substantial gains in $F / B$.

The effect on the spectra of different levels of filtration is shown in Fig. 7. In this picture, the spectra filtered with 47 and $157 \mu \mathrm{m}$ thick absorbers of $\mathrm{Rh}$ (corresponding to attenuation of 50 and $90 \%$ at 22.1 $\mathrm{keV}$, respectively) are shown along with the original spectrum.

To complement the data shown in Fig. 6, the gain in $F / B$ as a function of the thickness of the absorbing material was also assessed. The results, in terms of $F / B$ versus efficiency (at $22.1 \mathrm{keV}$ ) for selected materials are presented in Fig. 8. The term 'efficiency' is used to quantify the filter's attenuation, and is given by the fraction of photons in the $K_{\alpha}$ bin that are transmitted through the filter. This plot shows that, up to a certain value, increasingly thick filters enhance the observed $F / B$. Above this 'optimum' thickness, the reduction in the bremsstrahlung tail no longer compensates for the number of peak photons that are also being absorbed. The maximum values of $F / B$, together with the corresponding loss in efficiency and filter thickness are shown in Table 1 for different materials.

\subsection{Application to spectra produced in molybdenum and tungsten targets}

The general method introduced in the previous sec-

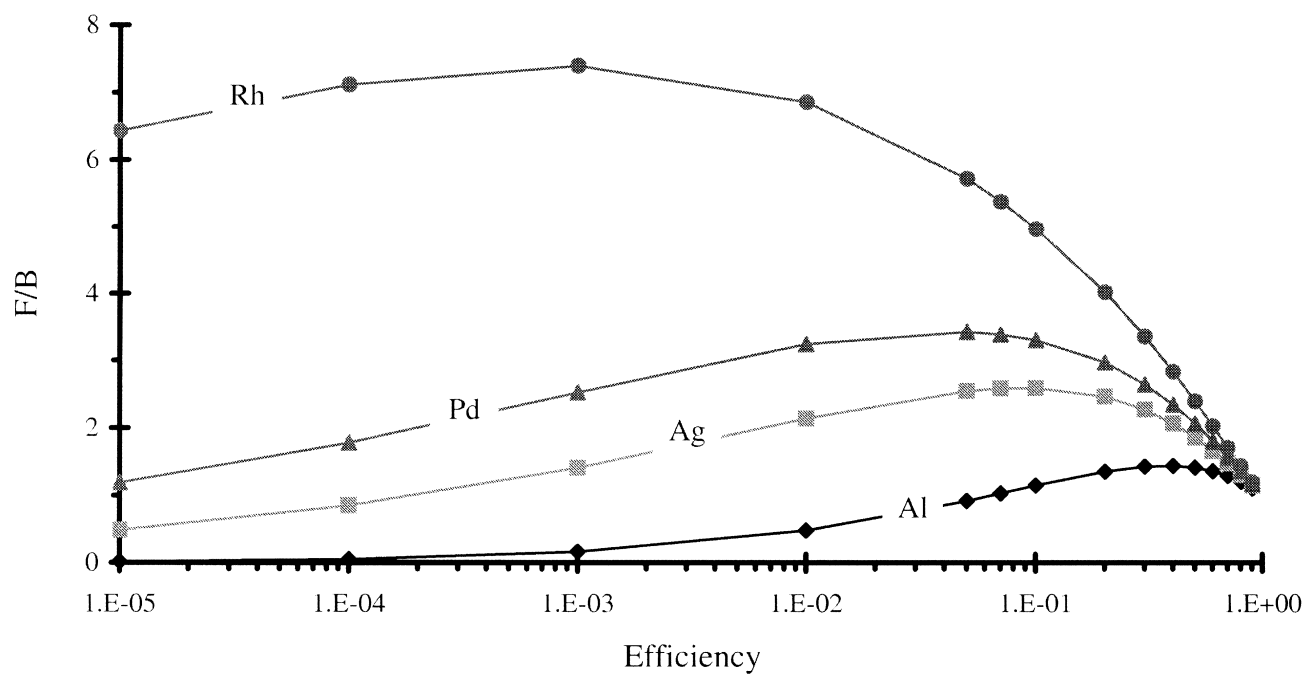

Fig. 8. Dependence of $F / B$ on the efficiency of the system, for different materials filtering an $A g$ spectrum. The value of $F / B$ has been normalised to that in the original spectrum while efficiency represents the fraction of $22.1 \mathrm{keV}$ photons transmitted through the filter. 
Table 1

Calculated values of $F / B$ versus efficiency for different filter materials applied to Ag spectra. The values of $F / B$ are normalised to that in the original spectrum, and efficiency represents the percentage of $K_{\alpha}$ photons that are transmitted through the absorber

\begin{tabular}{lccc}
\hline Material & Thickness $(\mu \mathrm{m})$ & $F / B$ & Efficiency $(\%)$ \\
\hline $\mathrm{Al}$ & 1370 & 1.44 & 40 \\
$\mathrm{Cu}$ & 40 & 1.42 & 40 \\
$\mathrm{Rh}$ & 470 & 7.40 & 0.1 \\
$\mathrm{Pd}$ & 228 & 3.43 & 5 \\
$\mathrm{Ag}$ & 160 & 2.60 & 10 \\
$\mathrm{Sn}$ & 102 & 1.62 & 30 \\
\hline
\end{tabular}

tion has also been applied to spectra generated with molybdenum (Mo) and tungsten (W) targets. The spec- tra used were calculated using the general models described and benchmarked by Birch and Marshall (1979) and Birch et al. (1979) and are presented in Fig. 9(a) and (b). The setup consisted of targets in reflection geometry (with a $12^{\circ}$ target), with the tubes operated at 35 and $150 \mathrm{kVp}$ for Mo and $\mathrm{W}$, respectively. Firstly, and following the method described in Section 3.1, the improvements in $F / B$ obtained with different filter materials have been determined. As previously, the results obtained show that the best filter materials to be employed are those whose $K$-edge lie just above their respective $K_{\alpha}$ lines. For spectra produced in Mo targets $\left(E_{K_{x}}=17.47 \mathrm{keV}\right)$, zirconium ( $\mathrm{Zr}$, $Z=40, K$-edge: $17.99 \mathrm{keV}$ ) represented the best solution while for spectra from W $\left(E_{K_{\alpha}}=59.32 \mathrm{keV}\right)$ thulium (Tm, $Z=69, K$-edge: $59.39 \mathrm{keV}$ ) was the material that optimised $F / B$.
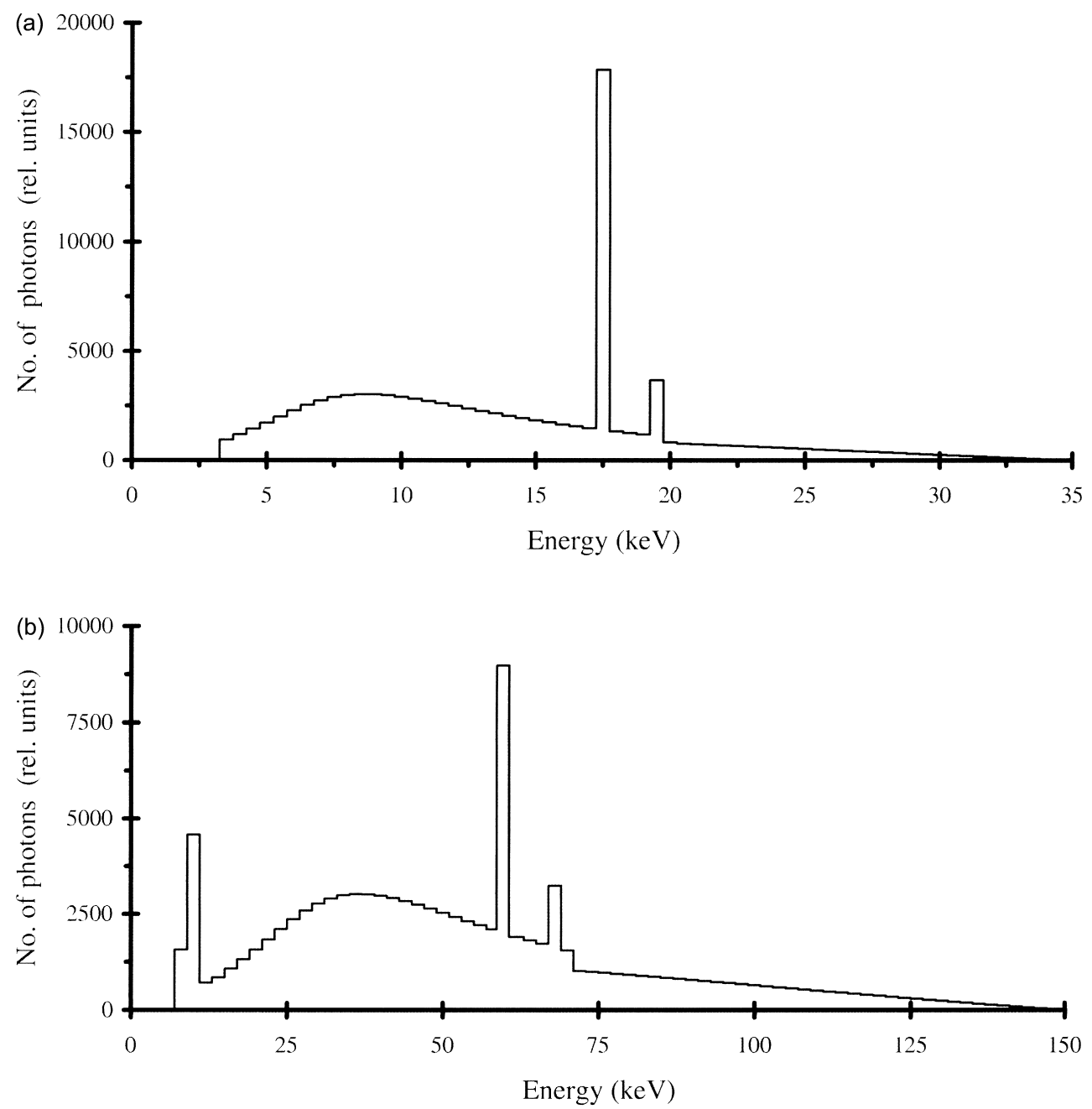

Fig. 9. Calculated X-ray spectra produced in reflection tubes with (a) Mo and (b) W targets, operated at 35 and $150 \mathrm{kVp}$, respectively. The setups consisted of a $12^{\circ}$ anodes, and the data was calculated using semiempirical models. 

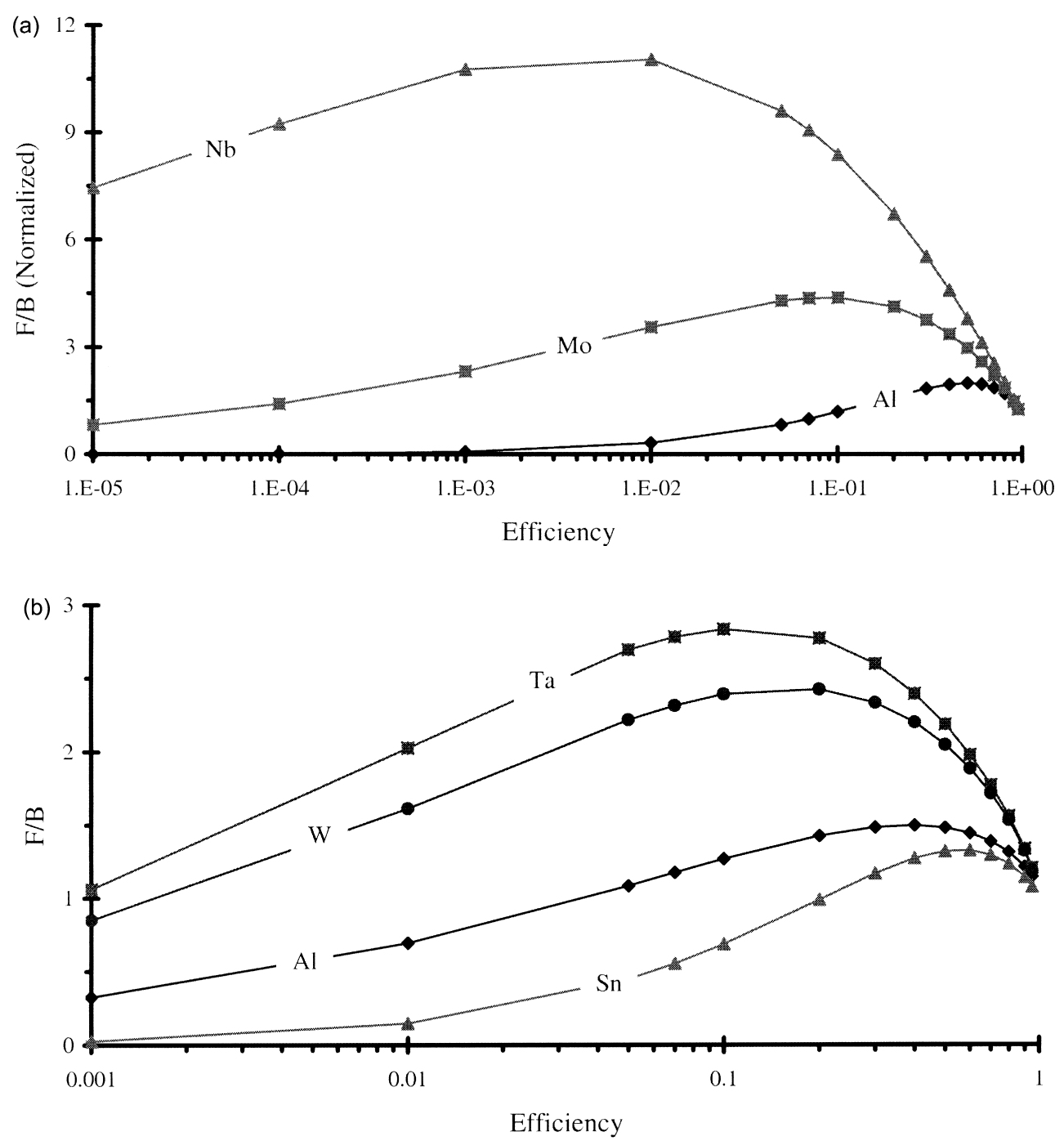

Fig. 10. Dependence of $F / B$ on the efficiency of the system, for different materials filtering (a) Mo and (b) W spectra. As in Fig. 8, the values and $F / B$ plotted are normalised to those in the original spectrum and the efficiency represents the fraction of $K_{\alpha}$ photons transmitted through the filter.

However, $\mathrm{Zr}$, Tm as well as well as elements with $70 \leq Z \leq 72$ are not suitable for this purpose as they are either flammable or noxious materials. For this reason, these elements have not been considered in this analysis and so we suggest niobium $(\mathrm{Nb}, Z=41, K$ edge: $18.98 \mathrm{keV})$ and tantalum (Ta, $Z=73, K$-edge: $67.42 \mathrm{keV})$ as the most suitable materials to filter Mo and $\mathrm{W}$ spectra, respectively.

The suitability of selected materials to filter Mo and W spectra, in terms of improvements in $F / B$ for a given attenuation at the $K_{\alpha}$ line, is shown in Fig. 10(a) and (b) for Mo and W, respectively. The 'optimum' thicknesses of different absorbers, maximum values of $F / B$ and corresponding loss in efficiency for these spectra are shown in Table 2 and Table 3.
Table 2

Calculated values of $F / B$ versus efficiency for different filter materials applied to the $35 \mathrm{kVp}$ Mo spectrum presented in Fig. 9(a). The values of $F / B$ are normalised to that in the original spectrum, and efficiency corresponds to the percentage of $K_{\alpha}$ photons transmitted through the absorber

\begin{tabular}{lccc}
\hline Material & Thickness $(\mu \mathrm{m})$ & $F / B$ & Efficiency $(\%)$ \\
\hline $\mathrm{Al}$ & 520 & 1.47 & 40 \\
$\mathrm{Cu}$ & 21 & 1.44 & 40 \\
$\mathrm{Nb}$ & 320 & 7.50 & 0.1 \\
$\mathrm{Mo}$ & 142 & 4.16 & 1 \\
$\mathrm{Ag}$ & 44 & 2.63 & 10 \\
$\mathrm{Sn}$ & 40 & 2.02 & 40 \\
\hline
\end{tabular}


Table 3

Calculated values of $F / B$ versus efficiency for different filter materials applied to the $150 \mathrm{kVpW}$ spectra presented in Fig. 9(b). The values of $F / B$ have been normalised to that in the original spectrum, with efficiency corresponding to the percentage of $K_{\alpha}$ photons transmitted through the absorber

\begin{tabular}{lccc}
\hline Material & Thickness $(\mu \mathrm{m})$ & $F / B$ & Efficiency $(\%)$ \\
\hline $\mathrm{Al}$ & 12500 & 1.50 & 40 \\
$\mathrm{Cu}$ & 385 & 1.51 & 40 \\
$\mathrm{Ag}$ & 82 & 1.41 & 0.1 \\
$\mathrm{Sn}$ & 103 & 1.32 & 60 \\
$\mathrm{Ta}$ & 377 & 2.83 & 1 \\
$\mathrm{~W}$ & 220 & 2.42 & 10 \\
$\mathrm{~Pb}$ & 122 & 1.67 & 30 \\
\hline
\end{tabular}

\section{Performance of different Ag-based transmission tubes}

The characteristics of spectra from transmission X-ray tubes with Ag-targets have also been assessed. Different target thicknesses, tube voltages and substrate materials have been considered. As previously, transmitted spectra were evaluated in terms of efficiency and the ratio of fluorescence to bremsstrahlung radiation.

\subsection{Target thickness and substrate material}

The setup considered in these simulation studies is similar to the one presented in Fig. 1. The configurations chosen consisted of a $2.2 \mu \mathrm{m} \mathrm{Ag}$ target deposited onto a $680 \mu \mathrm{m}$ AlN substrate, and the three configurations discussed in Section 2.2, i.e., $40 \mathrm{~nm}$ films deposited onto $\mathrm{Li}, \mathrm{B}$ and AlN substrates $(5 \mathrm{~mm}$, $1 \mathrm{~mm}$ and $680 \mu \mathrm{m}$ thick, respectively). In this case, $F / B$ can be plotted as a function of the number of $K_{\alpha}$ photons (which, in the simulated data, can easily be logged) reaching a $1.5 \mathrm{~cm}$ diameter detector positioned $15 \mathrm{~cm}$ away from the tube. In several applications the $K_{\alpha}$ line is actually used as the probing signal, so its yield may somehow be understood to represent the 'efficiency' of the X-ray tube. Again, since we are dealing with simulated data, $F / B$ was defined as the ratio of the number of $K_{\alpha}$ photons to the rest of the photons counted in the spectrum. All spectra were normalized to the same number of incident electrons, so that absolute values could be compared. Furthermore, and following the results presented in Section 3.1, different thicknesses of $\mathrm{Rh}$ were added to filter the transmitted spectra (thus varying the number of $K_{\alpha}$ photons reaching the detector).

The results obtained are shown in Fig. 11 and several conclusions can be inferred. At first glance it can be seen that thicker targets are not only more efficient but their spectra exhibit higher fluorescence to bremsstrahlung ratios. Since spectra emerging from thinner $\mathrm{Ag}$ targets actually exhibit higher $F / B$ (Távora et al., 1999a), this indicates that the bremsstrahlung produced in the substrate of thin Ag films degrades the spectral purity, and not even suitable filters seem to 'clean' them up. Therefore in applications requiring a film substrate, it seems more reasonable to use thicker $\mathrm{Ag}$ targets. With no added filtration (corresponding to the far right end of each curve) thick targets are $\approx 40$ times more efficient, an aspect that has been already

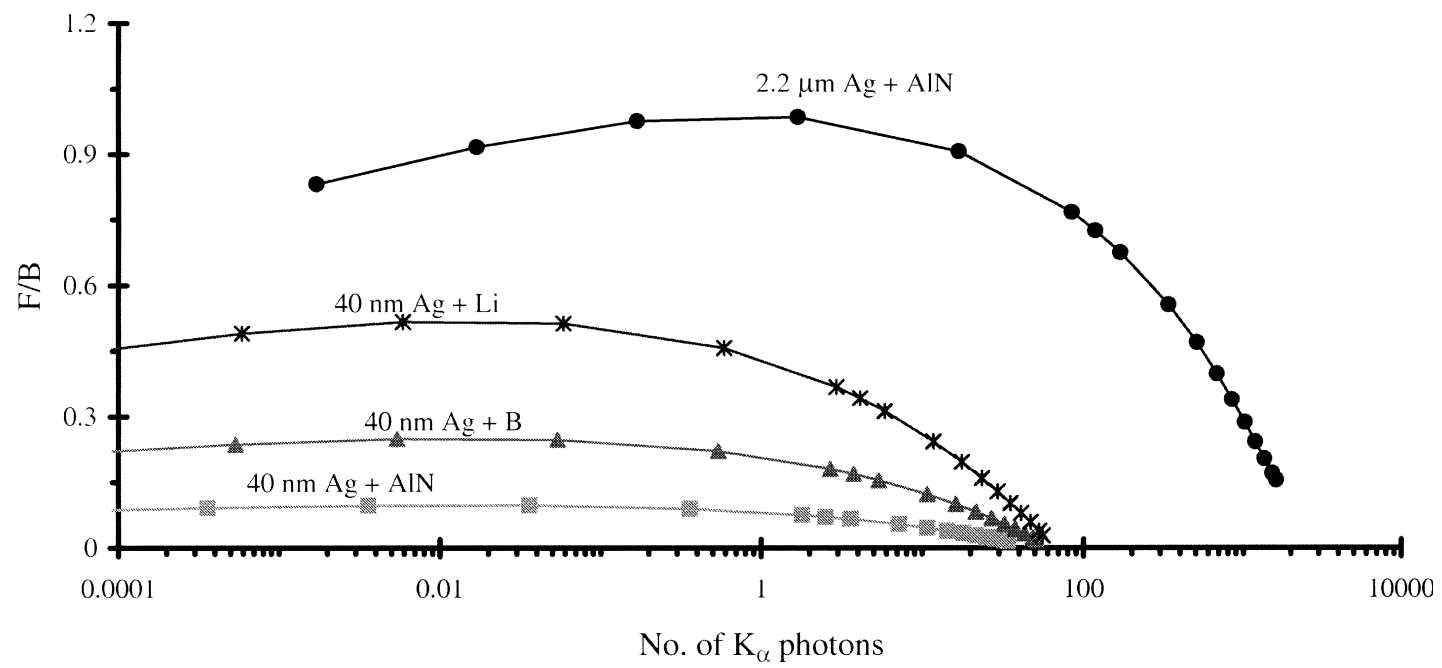

Fig. 11. Dependence of $F / B$ on the number of $K_{\alpha}$ photons detected, for different Ag-target transmission X-ray tubes. The configurations chosen were a $2.2 \mu \mathrm{m}$ target deposited onto a $680 \mu \mathrm{m}$ AlN substrate, and $40 \mathrm{~nm}$ targets deposited onto AlN, B and Li substrates. 
analysed in a previous communication (Távora et al., 1999a). The small differences observed in the efficiencies of the $40 \mathrm{~nm}$ target tubes can be understood on the grounds of the different attenuation of $22.1 \mathrm{keV}$ photons in the substrate; in these conditions (with no added filtration) it can be seen that $F / B$ is pretty much the same for these three configurations.

Finally filtered $\mathrm{Ag}$ spectra at maximum $F / B$ are shown in Fig. 12(a) and (b). These data correspond to spectra obtained from $2.2 \mu \mathrm{m}$ and $40 \mathrm{~nm}$ thick Ag targets deposited onto a $680 \mu \mathrm{m} \mathrm{AlN}$ and $5 \mathrm{~mm} \mathrm{Li}$ sub- strates, and filtered with 470 and $610 \mu \mathrm{m}$ slabs of $\mathrm{Rh}$, respectively. The spectra are normalised to the same total number of photons. Quite noticeable is an enhanced bremsstrahlung tail in the spectrum emerging from the setup comprising the thinner target. As introduced previously, the reason for this, and consequently the smaller $F / B$ shown in Fig. 11, is the considerable amount of bremsstrahlung produced in the substrate. As discussed Section 2, the bremsstrahlung yield increases with $Z$, so there is more bremsstrahlung produced in the thicker Ag target than in the Li substrate,
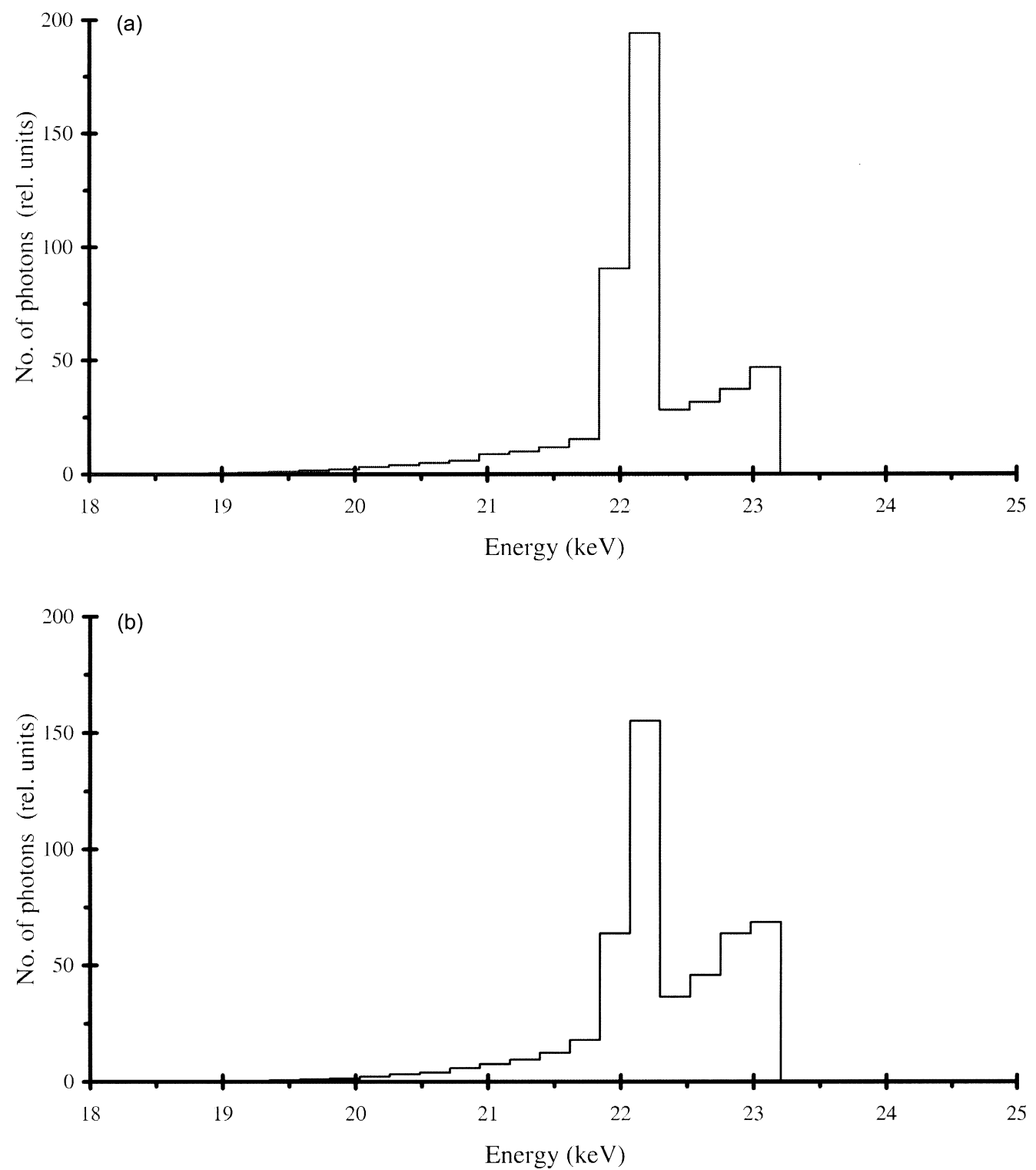

Fig. 12. Filtered spectra obtained from (a) a $2.2 \mu \mathrm{m} \mathrm{Ag} \mathrm{target} \mathrm{deposited} \mathrm{onto} \mathrm{a} 680 \mu \mathrm{m}$ AlN substrate and (b) a $40 \mathrm{~nm} \mathrm{Ag} \mathrm{film}$ deposited onto a $5 \mathrm{~mm} \mathrm{Li}$ substrate. The original spectra were filtered $\mathrm{Rh}$ slabs, and the data sets have been normalised to the same total number of photons. 


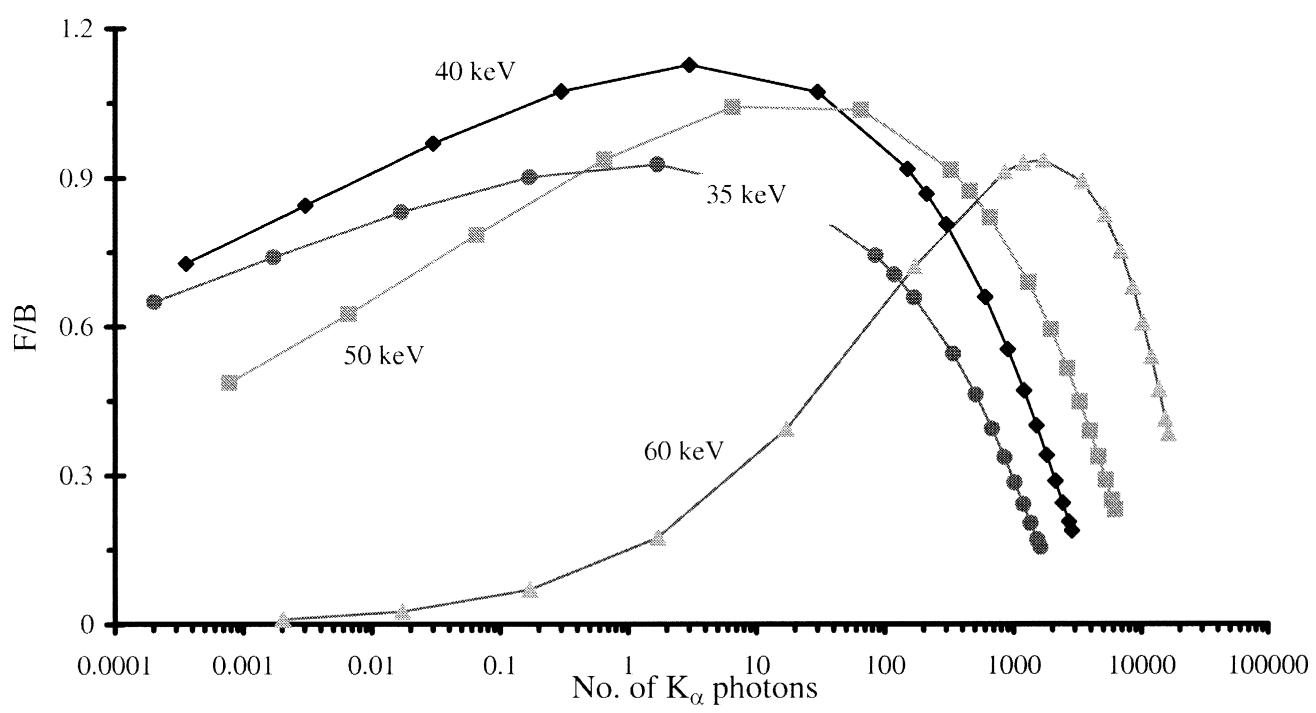

Fig. 13. Dependence of $F / B$ on the on the number of $K_{\alpha}$ photons detected, for Ag targets operated at different energies. The quantities shown have been calculated as those presented in Fig. 11.

but the production of fluorescence radiation in the former results in higher ratios of $F / B$. The fact that the number of photons in the filtered spectrum increases with energy up to $\approx 23.3 \mathrm{keV}$ is just a consequence of the decrease in the filter's photoelectric cross section up to its $K$-edge.

\subsection{Tube voltage}

In these studies we also considered the kind of setup presented in Fig. 1, but with 2.2, 3, 4.5 and $6 \mu \mathrm{m}$ thick Ag targets deposited onto a $680 \mu \mathrm{m}$ thick AlN sub- strate. The tubes were then considered to be operated at $35,40,50$ and $60 \mathrm{kV}_{\mathrm{p}}$, respectively. As discussed in Távora et al. (1999b), the thickness of the Ag films was chosen to match the situation where the production of bremsstrahlung radiation is maximized. In this case no enhancements in $F / B$ are obtained via the suppression of photon production. The transmitted spectra were then filtered with slabs of $\mathrm{Rh}$, and the results are presented in Fig. 13, where (absolute) efficiencies and $F / B$ were calculated as described in Section 4.1.

Several conclusions can be inferred from the data

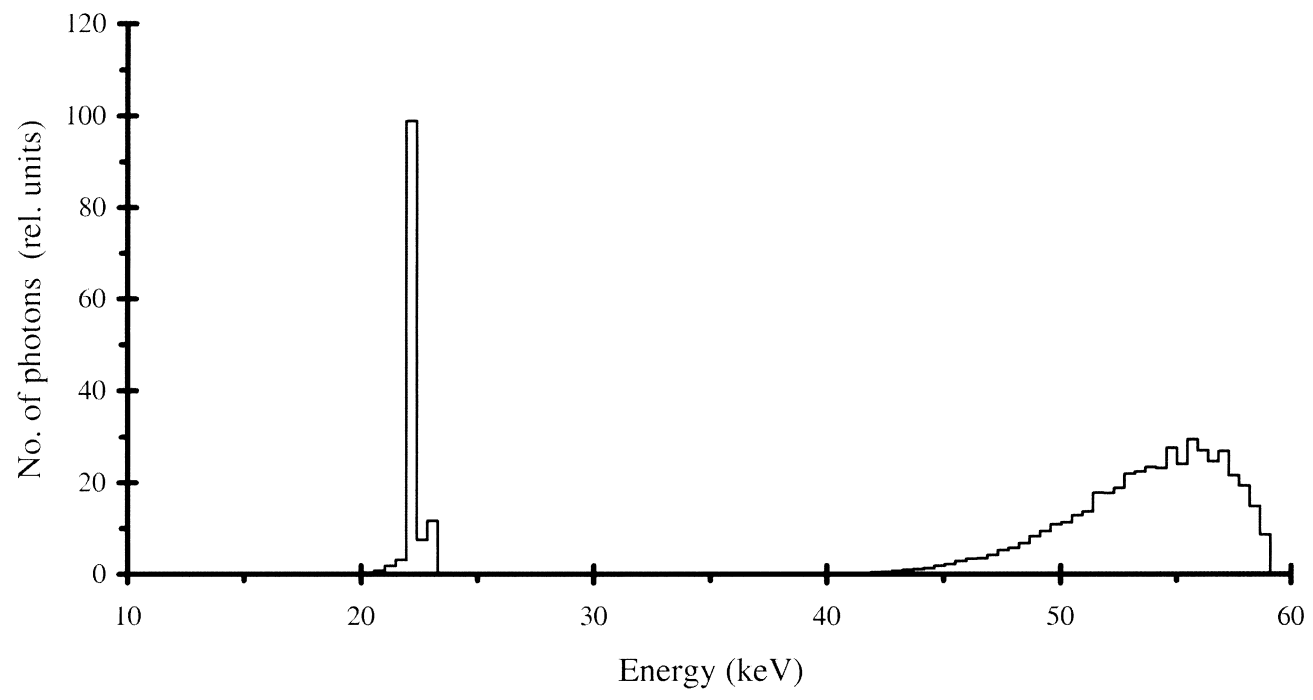

Fig. 14. Filtered spectrum obtained from a $6 \mu \mathrm{m} \mathrm{Ag} \mathrm{target} \mathrm{deposited} \mathrm{onto} \mathrm{a} 680 \mu \mathrm{m}$ AlN substrate. The system was considered to be operated at $60 \mathrm{kV}_{\mathrm{p}}$ and the output spectrum was filtered with a $630 \mu \mathrm{m}$ thick slab of $\mathrm{Rh}$. 
presented in Fig. 13. First, as discussed by Távora et al. (1999b), and briefly mentioned above, spectra obtained with higher tube voltages (the far right end of the curves) exhibit both higher $F / B$ and efficiencies. However, when filtration is added lower energy spectra show enhanced $F / B$ ratios, that can even exceed those from higher tubes voltages. The sudden drop in higher energy spectra's $F / B$ as thicker filters are used is due to the fact that a high energy bremsstrahlung tail starts to appear. This happens so because these photons are less attenuated in the filter, as Fig. 14 clearly shows. The same kind of behaviour was observed in spectra produced in Mo and $\mathrm{W}$ targets.

In the light of the data just presented one can conclude that the tube voltage is also of some importance when filtering is considered. In fact, although higher energy spectra are 'naturally' more efficient and with higher $F / B$ ratios, it might be more effective to filter lower energy spectra in order to obtain a more 'clean' spectrum. However, this improvement is obtained at the expense of efficiency.

\section{Conclusions}

The use of X-ray tubes as source providers in imaging application is nowadays a well-established approach. This paper focuses on methods to improve the ratio of fluorescence to bremsstrahlung radiation $(F / B)$ in X-ray tube spectra, and thus contributes to the development of 'quasi-monoenergetic' X-ray tubes. Firstly, the EGS4/GOS code was used in studies that aimed to understand the relevance of the substrate material in thin target applications. The results described show that the fraction of bremsstrahlung radiation produced in the tube substrate can be significantly reduced when low $Z$ materials are in use. It has however been observed that these materials also tend to be more transparent to the radiation produced in the target, especially at lower photon energies. This aspect may increase the fraction of bremsstrahlung photons transmitted through the system, thus reducing $F / B$ in the output spectrum. To overcome this problem, a suitable filter, that acts to remove the bremsstrahlung with no significant reduction in the fraction of fluorescence radiation, should be considered.

The choice of materials that should be employed to filter X-ray tube spectra with the aim of increasing the ratio of fluorescence to bremsstrahlung radiation was then discussed. The results presented for Mo, Ag, and W spectra show that materials whose $K$-edge lies just above the target's $K_{\alpha}$ line are the ones that, for a given attenuation at the $K_{\alpha}$ energy (i.e., a loss in efficiency), are more effective in increasing fluorescence to bremsstrahlung ratios. The data presented here show that, up to a certain thickness of filter material, $F / B$ does increase for more absorbing filters. However, above that thickness the opposite behaviour is observed. This suggests that the reduction in the number of fluorescence photons in the filters is no longer compensated by the absorption of photons from the bremsstrahlung tail. The values of 'optimum filter thicknesses' for different filter materials are presented, for $\mathrm{Mo}, \mathrm{Ag}$ and W tube spectra, in Tables 1-3.

Finally, the performance of Ag-target transmission $\mathrm{X}$-ray tubes with different configurations has been analyzed. It has been shown that, for the selected configurations, setups with thicker targets are not only more efficient but the spectra produced exhibit higher fluorescence to bremsstrahlung ratios. This is a direct consequence of the considerable production of bremsstrahlung radiation in the target substrate, even when low $Z$ materials like $\mathrm{Li}$ are considered. Therefore, for Ag tubes operating at the regimes considered, thick targets should be used, unless it is feasible to operate a thin target with no substrate. The dependence on the tube voltage was also studied, leading to the conclusion that, in order to maximise the ratio of fluorescence to bremsstrahlung radiation, it is more effective to use lower tube voltages.

In future, we shall continue our work in this area. Different models and cross section databases for the production of radiation in tube targets will be investigated. The updated Monte Carlo codes will then be used to simulate the response of diagnostic X-ray tubes, and the results compared with experimental data. We also expect to carry out further design studies that aim at understanding the production of radiation in X-ray tubes leading, when possible, to the optimization of these sources.

\section{Acknowledgements}

This work was carried out in Departamento the Física da Universidade de Coimbra (Research Unit No. 217/94) and the Physics Department of the University of Surrey. L.M.N. Távora and E.J. Morton acknowledge financial support from Fundação para a Ciência e Tecnologia (Portugal) through Programs PRAXIS XXI/BPD/18870/98 and PRAXIS XXI/3/3.1/ CTAE/1920/95, and the National Radiological Protection Board, Chilton, Oxon, UK.

\section{References}

Andreo, P., 1991. Monte Carlo techniques in medical radiation physics. Phys. Med. Biol. 36, 861.

Archer, B.R., Wagner, L.K., 1998. Determination of diagnostic X-ray spectra with characteristic radiation using attenuation analysis. Med. Phys. 15, 637. 
Beaman, S.A., Lillicrap, S.C., 1982. Optimum X-ray spectra for mammography. Phys. Med. Biol. 27, 1209.

Berger, M.J., Hubbell, J.H., 1987. XCOM: photon cross sections on a personal computer. National Bureau of Standards Report 87-3757.

Bhat, M., Pattison, J., Bibbo, G., Caon, M., 1998 a. Diagnostic X-ray spectra: a comparison of spectra generated by different methods with a measured spectrum. Med. Phys. 25, 114.

Bhat, M., Pattison, J., Bibbo, G., Caon, M., 1998b. Off-axis X-ray spectra: a comparison of Monte Carlo simulated and computed X-ray spectra with measured spectra. Med. Phys. 26, 303.

Birch, R., Marshall, M., 1979. Computation of bremsstrahlung X-ray spectra and comparison with spectra measured with a Ge(Li) detector. Phys. Med. Biol. 24, 505.

Birch, R., Marshall, M., Ardran, G.M., 1979. Catalogue of Spectral Data for Diagnostic X-rays. The Hospital Physicists' Association, London.

Bissonete, J.P., Schreiner, L.J., 1992. A comparison of semiempirical models for generating tungsten target X-ray spectra. Med. Phys. 19, 579.

Boone, J.M., Seibert, A.S., 1997. An accurate method for computer-generating tungsten anode X-ray spectra from 30 to $140 \mathrm{kV}$. Med. Phys. 24, 1661.

Boyanov, B., 1996. Subroutine MUCAL. C version 1.2. Code and documentation available from ftp://ixs.csrri.iit.edu/ programs/mcmaster.

Briesmeister, J.F., 1997. MCNP ${ }^{(m)}$ - a general Monte Carlo $n$-particle transport code. Los Alamos National Laboratory Report LA-12625-M.

Jauch, J.M., Rohrlich, F., 1976. The Theory of Photons and Electrons, 2nd expanded edition. Springer, Heidelberg.

Jenkins, T.M., Nelson, W.R., Rindi, A., 1988. Monte Carlo Transport of Electrons and Photons. Plenum Press, New York.

Kramers, H.A., 1920. On the theory of X-ray absorption and of the continuous X-ray spectrum. Philos. Mag. 46, 836.

Martin, C.J., Sutton, D.G., Sharp, P.F., 1999. Balancing patient dose and image quality. Appl. Rad. Isot. 50, 1.

Massoumzadeh, P., Rudin, S., Bednarek, D.R., 1998. Filter material selection for region of interest radiologic imaging. Med. Phys. 25, 161.

McMaster, W.H., Del Grande, N.K., Mallet, J.H., Hubbell,
J.H., 1969. Compilation of X-ray cross sections. Lawrence Livermore Radiation Laboratory Report UCRL 50174.

Nelson, W.R., Hirayama, H., Rogers, D.W.O., 1985. The EGS4 code system. Stanford Linear Accelerator Report SLAC-265.

Nelson, W.R., Bielajew, A.F., Rogers, D.W.O., Hirayama, H., Namito, Y., 1997. EGS4 in '97: a decade plus of enhancements. In: Proceedings of the First International Workshop on EGS4 (Japan), KEK Proceedings 97, 16.

Perkins, S.T., Cullen, D.E., Seltzer, S.M., 1991. Tables and graphs of electron-interaction cross sections from $10 \mathrm{eV}$ to $100 \mathrm{GeV}$ derived from the LLNL evaluated electron data library (EEDL), $Z=1-100$. Lawrence Livermore National Laboratory Report UCRL-50400, vol. 31.

Rudin, S., Bednarek, D.R., 1993. Comparison of filter material and design for use in ROI angiography. Proc. SPIE 1896, 354.

Sandborg, M., Carlsson, C.A., Carlsson, G.A., 1994a. Shaping X-ray spectra with filters in X-ray diagnostics. Med. \& Biol. Eng. \& Comput. 32, 384.

Sandborg, M., Dance, D.R., Persliden, J., Carlsson, G.A., 1994b. A Monte Carlo program for the calculation of contrast, noise and absorbed dose in diagnostic radiology. Compu. Meth. and Prog. in Biomed. 42, 167.

Seltzer, S.M., 1991. Electron-photon Monte-Carlo calculations: the ETRAN code. Appl. Radiat. Isot. 24, 917.

Sundararaman, V., Prasad, M.A., Vora, R.B., 1973. Computed spectra from diagnostic and therapeutic X-ray tubes. Phys. Med. Biol. 18, 208.

Távora, L.M.N., Morton, E.J., 1998. Photon production using a low energy electron expansion of the EGS4 code system. Nucl. Instr. and Meth. B143, 253.

Távora, L.M.N., Morton, E.J., Gilboy, W.B., 1999a. Design considerations for transmission X-ray tubes operating at diagnostic energies. Journal of Physics D: Applied Physics, submitted.

Távora, L.M.N., Morton, E.J., Gilboy, W.B., 1999 b. Optimisation of transmission target X-ray tubes for imaging applications using Monte Carlo based methods. Presented at 44th SPIE Meeting, 18-23 July 1999, Denver, Colorado. Accepted for publication in the SPIE Conference Proceedings.

Tucker, D.M., Barns, G.T., Chakraborty, D.P., 1991. Semiempirical model for generating tungsten target X-ray spectra. Med. Phys. 18, 211. 